\title{
Comparative Analysis on Performance Evaluation of Naval Minesweeping
}

\author{
Huidong Lu1, a , Xinqin Chen ${ }^{2, b}$, Wei Zhang ${ }^{1}$, Chang Shu'1, Xuanmin Li2, \\ Longteng $\mathrm{Li}^{1}$, Chengwen Zhu ${ }^{1}$
}

\author{
${ }^{1}$ Dept. of Command and control, Wuhan Ordnance Officer School, Wuhan, Hubei, 430075, China \\ ${ }^{2}$ Dept. of Computer, Wuhan Bridge College, Wuhan, Hubei, 430052, China

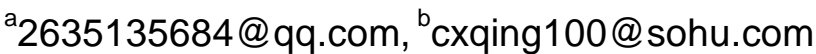

\begin{abstract}
Keywords: Markov process Bayesian estimation minesweeping probability remaining mine Abstract. The estimation of number of remaining mines is an important basis for the performance evaluation of naval minesweeping, and the accuracy of the estimation is related to the mastery of prior information and the selection of evaluation model. In this paper, we build the evaluation models by employing Markov process and Bayesian estimation theory to estimate the number of remaining mines. Then simulation experiments based on the above models were carried out, using the operational data which come from prior information of the minefield and information of swept mines. The results show that the Bayesian estimation is more reliable than Markov process in the case of sufficient prior information.
\end{abstract}

\section{Introduction}

The main purpose of mine operational evaluation is to quantify the risk of minefield after minesweeping, while the number of remaining mine is one of the key links for that. How to estimate the number of remaining mines according to sampling information has become the main problem which is most concerned about by various models. We can build a model which meets the following requirements: strong description ability which is related to mine array state, information retrieval ability, practical value for dealing with various problems, different processing methods to choose according to the different conditions [1-5]. In this paper, two typical models, Markov process and Bayesian estimation [4-7] are analyzed and compared. The estimation of remaining mine number is studied for different prior information, starting from the samples of information related the mines which have been swept.

\section{Establishment of the Model of Performance Evaluation}

\section{Mathematical Model Based on Markov process.}

Markov process is a random process which can describe many practical problems whose coming state is related only to current state. In order to describe it, we define some symbols as follows: $D C$-a mine will explode when the sweeper affected it $D C$ times, $Q$-maximal value of $D C, N$-sum of mine sweeping times, $x_{q}^{n}$-number of mine which $D C$ is $q$, swept for the nth time,

$\boldsymbol{X}_{n}=\left(x_{1}^{n}, \mathbf{L} x_{Q-1}^{n}, x_{Q}^{n}\right)^{T}$-vector made up of variables $x_{i}^{n}, p_{0}$-mine-sweeping probability, $y_{n}$-sum of the mine which have been swept, $\boldsymbol{Y}_{n}$-vector made up of variables $y_{n}, \boldsymbol{Y}_{n}=\left(y_{1}, \mathrm{y}_{2}, \mathrm{~L}, y_{n}\right)^{T}, w_{q}^{n}$ - variable which denotes error of $x_{q}^{n+1}, \boldsymbol{W}_{n}$ - vector made up of variables $w_{q}^{n}, e_{n}$ - variable which denotes error of $\mathrm{y}(\mathrm{n}), \boldsymbol{E}_{n}$ - vector made up of variables $e_{n}$.

According to Markov process theory [3, 4, 8], when the minefield has been swept $n+1$ times, the number of mines whose $D C$ is $q$ only relates to the mines whose $D C$ are $q$ and $q+1$. Then the equation can be obtained as follows: 
$x_{q}^{n+1}=\left(1-p_{0}\right) x_{q}^{n}+p_{0} x_{q+1}^{n}+w_{q}^{n}, n \in[1, N], q \in[1, Q]$

Suppose

$$
\boldsymbol{A}=\left[\begin{array}{cccccc}
1-p_{0} & p_{0} & 0 & \mathbf{L} & 0 & 0 \\
0 & 1-p_{0} & p_{0} & \mathbf{L} & 0 & 0 \\
\mathbf{L} & \mathbf{L} & \mathbf{L} & \mathbf{L} & \mathbf{L} & \mathbf{L} \\
0 & 0 & 0 & \mathbf{L} & p_{0} & 0 \\
0 & 0 & 0 & \mathbf{L} & 1-p_{0} & p_{0} \\
0 & 0 & 0 & \mathbf{L} & 0 & 1-p_{0}
\end{array}\right], \boldsymbol{C}=\left[\begin{array}{c}
p_{0} \\
0 \\
\mathbf{M} \\
\mathbf{M} \\
0 \\
0
\end{array}\right]
$$

The equation (1) can be rewritten as follows:

$$
\boldsymbol{X}_{n+1}=\boldsymbol{A} \boldsymbol{X}_{n}+\boldsymbol{W}_{n}=\mathrm{L}=\boldsymbol{A}^{n+1} \boldsymbol{X}_{0}+\boldsymbol{W}_{n}
$$

When the minefield has been swept $n+1$ times and the number of mines which have been swept out is $y_{n+1}$, it is only related to mines whose $D C$ is 1 :

$y_{n+1}=p_{0} x_{1}^{n}+e_{n+1}=\boldsymbol{C}^{\mathrm{T}} \boldsymbol{X}_{n}+e_{n+1}$

The above equation can be rewritten as follows:

$$
y_{n}=\boldsymbol{C}^{\mathrm{T}} \boldsymbol{X}_{n-1}+e_{n}=\boldsymbol{C}^{\mathrm{T}}\left(\boldsymbol{A}^{n-1} \boldsymbol{X}_{0}+\boldsymbol{W}_{n}\right)+e_{n}
$$

If

$$
\boldsymbol{H}_{n}=\left[\begin{array}{c}
\left(\boldsymbol{C}^{\prime}\right)^{T}\left(\boldsymbol{A}^{\prime}\right)^{1} \\
\left(\boldsymbol{C}^{\prime}\right)^{T}\left(\boldsymbol{A}^{\prime}\right)^{2} \\
\mathrm{M} \\
\left(\boldsymbol{C}^{\prime}\right)^{T}\left(\boldsymbol{A}^{\prime}\right)^{n-1}
\end{array}\right], \boldsymbol{Y}_{n}=\left[\begin{array}{c}
y_{1} \\
y_{2} \\
\mathrm{M} \\
y_{n}
\end{array}\right], \boldsymbol{E}_{n}=\boldsymbol{C}^{\mathrm{T}} \cdot \boldsymbol{W}_{n}+\left[\begin{array}{c}
e_{1} \\
e_{2} \\
\mathrm{M} \\
e_{n}
\end{array}\right]
$$

Then according to the equation (4), the following expression can be obtained:

$\boldsymbol{Y}_{n}=\boldsymbol{H}_{n} \boldsymbol{X}_{0}+\boldsymbol{E}_{n}$

\section{Mathematical Model based on Bayesian estimation theory}

Some symbols are defined as follows: $N$-the total number of mine, $\pi(m)$-the prior distribution of mine, $\mathbf{m}_{0}=\left[m_{10}, m_{20}, \mathrm{~L}, m_{n 0}\right]$ - the initial allocation proportion of the mine number when the minefield is divided into $\mathrm{n}$ regions, $\mathbf{p}_{j}=\left[p_{1 j}, p_{2 j}, \mathrm{~L} p_{n j}\right]^{T}$-the mine sweeping probability of the single mine in the sub area when the $j$ th estimation is done, $\mathbf{x}_{j}=\left[x_{1 j}, x_{2 j}, \mathrm{~L} x_{n j}\right]$-the number of mines in each sub region between $\mathrm{j}$ and $\mathrm{j}-1, R$ - the remaining number of mines of the current status, $X$ - the sample of swept mines in the current status, $N_{f}$ is the total number of swept mines..

Suppose $\mathbf{X}_{j}=\left[\mathbf{x}_{1}, \mathbf{x}_{2}, \mathrm{~L} \mathbf{x}_{j}\right]^{T}, N_{j}=\sum_{k=1}^{j} \sum_{i=1}^{n} x_{i j}, \Delta N_{j}=N_{j}-N_{j-1}, P_{j}=\mathbf{m}_{0} \times \mathbf{p}_{j}$

If the remaining number of mine is estimated for the first time, according to Bayesian statistical theory, the joint distribution of the sample $\left(X_{1}\right)$ and the number of remaining mines of the whole area $\left(R_{1}\right)$ is:

$h\left(R_{1}, \mathbf{X}_{1}\right)=\pi\left(R_{1}+N_{1}\right)\left(\begin{array}{c}R_{1}+N_{1} \\ N_{1}\end{array}\right) P_{1}^{N_{1}}\left[1-P_{1}\right]^{R_{1}}$

The conditional distribution of $R_{1}$ is:

$\pi\left(R_{1} \mid \mathbf{X}_{1}\right)=\pi\left(R_{1}+N_{1}\right) \frac{\left(R_{1}+N_{1}\right) !}{R_{1} !}\left[1-P_{1}\right]^{R_{1}} /\left[\sum_{R_{1}=0}^{N-N_{1}} \pi\left(R_{1}+N_{1}\right) \frac{\left(R_{1}+N_{1}\right) !}{R_{1} !}\left[1-P_{1}\right]^{R_{1}}\right]$

For sub area $i$, the distribution proportion of the number of remaining mines is:

$$
m_{i 1}=m_{i 0} \frac{1-p_{i 1}}{1-P_{1}}
$$


The posterior distribution of the number of remaining mines can be obtained:

$$
\pi\left(r_{i 1} \mid \mathbf{X}_{1}\right)=\sum_{R_{1}=r_{i 1}}^{N-N_{1}} \pi\left(R_{1} \mid \mathbf{X}_{1}\right)\left(\begin{array}{l}
R_{1} \\
r_{i 1}
\end{array}\right)\left(m_{i 1}\right)^{r_{i 1}}\left[1-m_{i 1}\right]^{R_{1}-r_{i 1}}
$$

Set $G_{1}=\sum_{R_{1}=0}^{N-N_{1}} \pi\left(R_{1}+N_{1}\right) \frac{\left(R_{1}+N_{1}\right) !}{N_{1} !}\left[1-P_{1}\right]^{R_{1}}$, we can get the following expressions:

$$
\begin{aligned}
\pi\left(r_{i 1} \mid \mathbf{X}_{1}\right) & =\frac{1}{G_{1}} \sum_{R_{1}=r_{11}}^{N-N_{1}}\left\{\pi\left(R_{1}+N_{1}\right) \frac{\left(R_{1}+N_{1}\right) !}{R_{1} !} \frac{R_{1} !}{\left(R_{1}-r_{i 1}\right) ! r_{i 1} !}\left[1-P_{1}\right]^{R_{1}}\left(m_{i 0} \frac{1-p_{i 1}}{1-P_{1}}\right)^{r_{i 1}}\left[1-m_{i 0} \frac{1-p_{i 1}}{1-P_{1}}\right]^{R_{1}-r_{i 1}}\right\} \\
= & \frac{1}{G_{1}} \sum_{R_{1}=r_{i 1}}^{N-N_{i}} \pi\left(R_{1}+N_{1}\right) \frac{\left(R_{1}+N_{1}\right) !}{\left(R_{1}-r_{i 1}\right) ! r_{i 1} !} \cdot\left[m_{i 0}\left(1-p_{i 1}\right)\right]^{r_{11}}\left[1-P_{1}-m_{i 0}\left(1-p_{i 1}\right)\right]^{R_{1}-r_{i 1}}
\end{aligned}
$$

We can see $\pi\left(R_{j-1} \mid \mathbf{X}_{j-1}\right)$ as the prior distribution in the $j$ th estimation, and obtain the distribution of number of remaining mines:

$$
\left\{\begin{array}{l}
\pi\left(R_{j} \mid \mathbf{X}_{j}\right)=\frac{1}{G_{j}} \pi\left(R_{j}+N_{j}\right) \frac{\left(R_{j}+N_{j}\right) !}{R_{j} !}\left[1-P_{j}\right]^{R_{j}} \\
\pi\left(r_{i j} \mid \mathbf{X}_{j}\right)=\frac{1}{G_{1}} \sum_{R_{j}=r_{i j}}^{N-N_{j}} \pi\left(R_{j}+N_{j}\right) \frac{\left(R_{j}+N_{j}\right) !}{\left(R_{j}-r_{i j}\right) ! r_{i j} !}\left[m_{i 0}\left(1-p_{i j}\right)\right]^{r_{i j}}\left[1-P_{j}-m_{i 0}\left(1-p_{i j}\right)\right]^{R_{j}-r_{i j}} \\
G_{j}=\sum_{R_{j}=0}^{N-N_{j}} \pi\left(R_{j}+N_{j}\right) \frac{\left(R_{j}+N_{j}\right) !}{N_{j} !}\left[1-P_{j}\right]^{R_{j}}
\end{array}\right.
$$

By analysis of equation (11), we find that the distribution of remaining number of mines has nothing to do with previous estimation. So it can be rewritten as follows:

$$
\left\{\begin{array}{l}
\pi(R \mid \mathbf{X})=\frac{1}{G} \pi(R+N) \frac{(R+N) !}{R !}[1-P]^{R} \\
\pi\left(r_{i} \mid \mathbf{X}\right)=\frac{1}{G} \sum_{R=r_{i}}^{N-N_{f}} \pi(R+N) \frac{(R+N) !}{\left(R-r_{i}\right) ! r_{i} !}\left[m_{i 0}\left(1-p_{i}\right)\right]^{r_{i}}\left[1-P-m_{i 0}\left(1-p_{i}\right)\right]^{R-r_{i}} \\
G=\sum_{R_{j}=0}^{N-N_{j}} \pi(R+N) \frac{(R+N) !}{N !}[1-P]^{R}
\end{array}\right.
$$

Then we can obtain the mean and variance of remaining mines as follows:

$$
\left\{\begin{array}{l}
E_{R}=\sum_{R=0}^{N-N_{f}} \pi\left(R \mid N_{f}\right) \cdot R \\
V_{R}=\sum_{R=0}^{N-N_{f}} \pi\left(R \mid N_{f}\right) \cdot\left(R-E_{R}\right)^{2}
\end{array}\right.
$$

\section{Sample Data Acquisition}

We can obtain the sample data from Monte Carlo stochastic simulation algorithm or the results of minesweeping training and see it as the observation sequence value of the swept mines. Here we take the result of Monte Carlo method simulation as the sample data:

Generate a random number $p_{i}, p_{i} \in[0,1]$ for each mine. Define $p_{0}^{\prime}$ as the threshold valve. If $p_{i}<p_{0}^{\prime}$, the mine can be considered swept mine and taken as the observation sequence value. 


\section{Emulation and results}

Assume that there are 40 mines in one minefield and the maximum value of $D C$ is 4 . Its distribution is [15;15;5;5]. The minesweeping probability is 0.6 and 0.9 in Markov process model. If the quality of prior information is high, the estimation result which comes from binomial distribution is better than others in Bayesian estimation model. So we adopt binomial distribution: $B(50,0.8)$ and $B(80,0.5)$. Take the emulation 30 rounds ( $N=4$ times per round). The estimated values of the remaining mines in above mine field are plotted in Fig1, Fig2.

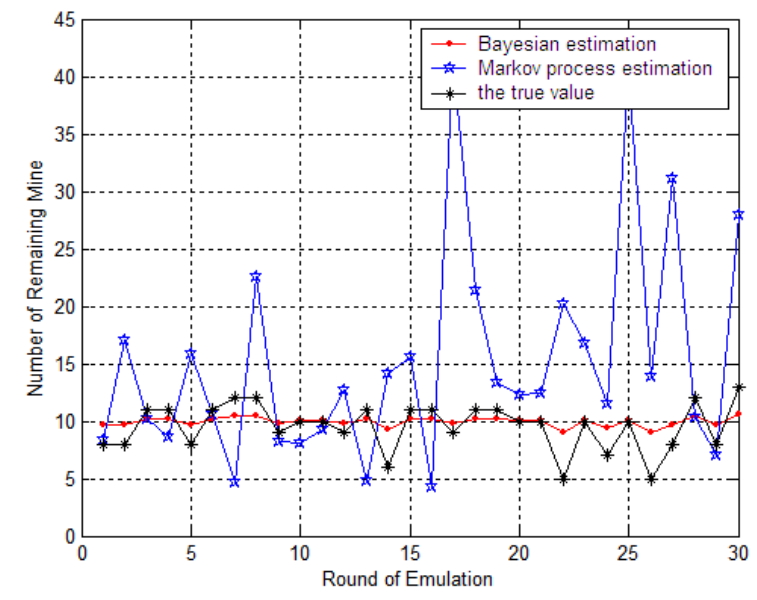

Fig1.a number of remaining mines when $B(80,0.5), P_{0}=0.9$

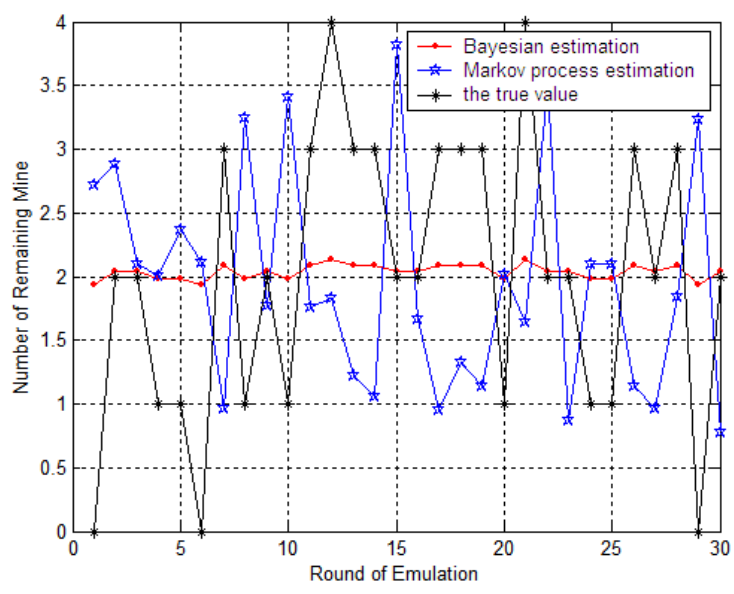

Fig2.a number of remaining mines when $B(80,0.5), P_{0}=0.6$

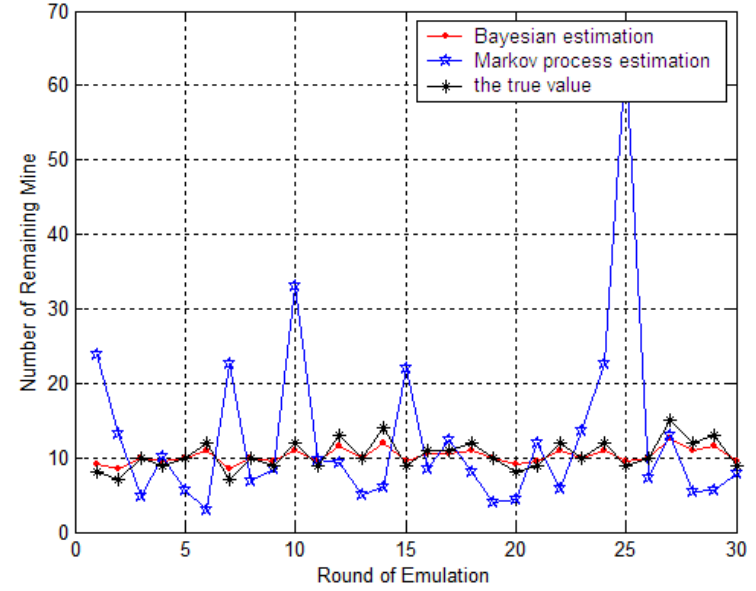

Fig1.b number of remaining mines when $B(50,0.8), P_{0}=0.9$

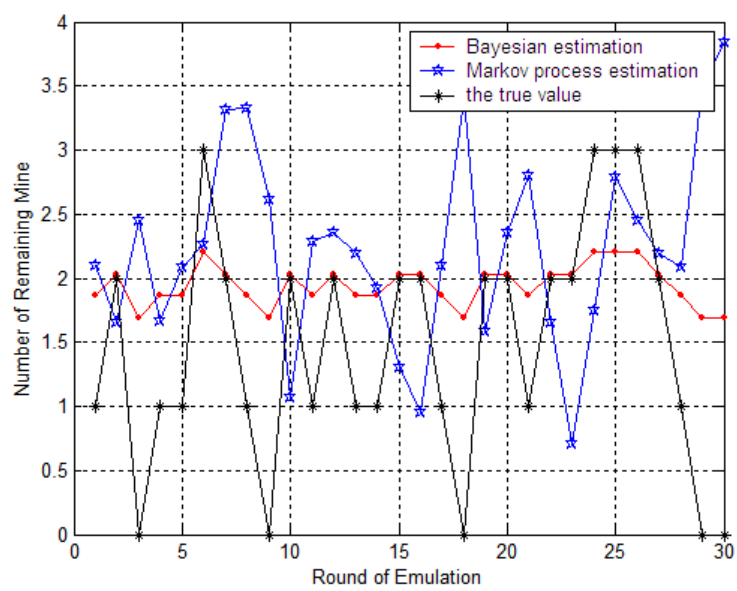

Fig2.b number of remaining mines when $B(50,0.8), P_{0}=0.6$

In Fig1.a and Fig2.a, the minesweeping probability is 0.9 and 0.6 , and the prior distribution is binomial distribution $B(80,0.5)$. In order to make the prior information closer to the truth, we change the parameters and take $B(50,0.8)$ as the prior distribution. Then the estimation results can be obtained as Fig2.a and Fig2.b.

\section{Discussions and Conclusions}

The result shows that Bayesian estimation model is better than Markov process model. Although Markov process model can reflect the statistical results, for some round of simulations, the deviation will appear between the estimated result and the true value. On the contrary, the trend of the curve is 
consistent with the true value in Bayesian estimation model, although there are different amplitudes with the different prior information. The results are achieved because the Bayesian estimation model can exploit the prior information fully which can be obtained accurately in many practical situations.

Comparing fig. 1 with fig. 2 , we find that the probability has a direct influence on the assessment. The high sweeping probability can obtain satisfactory results because the sampling data is affected to a lesser degree by the "noise". In both fig.1b and fig. $2 a$, we can find that the amplitude of the posterior estimate has been greatly improved compared with the situation shown in Fig.1a and fig.2b because the parameter $P_{b}=(0.8,0.5)$ in the latter is closer to weeping probability $P_{0}=(0.9,0.6)$. This shows that when we use the Bayesian method to estimate the number of remaining mines, the accuracy of prior information will play a crucial role.

Of course, it is feasible to adopt Markov process model only if we cannot get prior information, moreover, Markov process model can process more complicated situation. So in practice, the choice of what kind of model depends on the availability of information on the situation.

\section{References}

[1] Xinqin Chen, Huidong Lu, Longteng Li, Chang Shu. Performance Evaluation of Naval Minesweeping and Estimation of the Number of Remaining Mines based on Bayesian Estimation Theory. IFCEM2015: 451-455

[2] Chen Xin-qin, Lu Hui-dong, Zhu Cheng-wen.Performance Evaluation of Naval Minesweeping and Estimation of the Number of Remaining Mines Based on Markov Process. $\operatorname{ETEEEM(2014):739-742~}$

[3] Chen Xin-qin, Li Longteng, Li Xuanmin.Research on the evaluation model of effect of naval minesweeping operation in complex conditions. ETEEEM (2014):743-747

[4] Chen Xin-qin. Study on Pivotal Techniques of Mine Sweeping Based on Magnetic Fuse Mine [D]. Wuhan: Naval University of Engineering. 2009.

[5] Wu Xi-zhi.Bayesian statistics [M]. Beijing: China Statistics Press, 2000.

[6] Jia Nai-guang.Statistical Decision deals with Bayesian analysis. Beijing: China Statistics Press , 2001.

[7] Mao Shi-song. Bayesian statistics [M]. Beijing: China Statistics Press , 1999.

[8] Zhang xu. Study on the operation of anti mine warfare for mine-hunting ship [D]. Dalian : Dalian Naval Academy Press. 2005. 